\title{
Clinical Features of Demyelinating Lesion in Medulla Oblongata and Cervical Cord Junction With the First Symptom of Neck Pain Misdiagnosed as Acute Stroke
}

\author{
Yi Bao a, b, Xingyue Li ${ }^{\mathrm{a}}$, Yajie $\mathrm{Hu}^{\mathrm{a}}$, Lixia Qin ${ }^{\mathrm{a}}$, Miao Zhanga, \\ Ying Wang ${ }^{\mathrm{a}}$, Guangjian Liu ${ }^{\mathrm{a}}$
}

\begin{abstract}
This article aims to describe the clinical features of a demyelinating lesion in the medullary and cervical spinal cord junctions with a first symptom of neck pain misdiagnosed as acute stroke. The patient was initially misdiagnosed as ischemic stroke through stroke-like onset, clinical manifestations, physical examination, and brain computed tomography (CT); but the patient's symptoms were not completely consistent with typical physical examination of stoke. Combined with brain magnetic resonance imaging (MRI) which showed demyelinating lesions and diagnostic treatment, the final diagnosis was the demyelinating lesion of the medulla oblongata and cervical cord junction. Acute hemiplegia in quiet state is not necessarily a stroke. Detailed physical examination and imaging data are helpful for differential diagnosis. Diagnostic treatment can help to confirm the diagnosis if necessary. Early use of methylprednisolone shock therapy combined with intravenous administration of gamma globulin for demyelinating disease can achieve good results, save limb function; and long-term medication is required to prevent rebound.
\end{abstract}

Keywords: Demyelination; Acute stroke; IVIG; Cerebrospinal fluid

\section{Introduction}

Demyelinating disease is a kind of acquired central nervous system disease with unknown etiology, different clinical manifestations and similar characteristics, mostly caused by immune abnormalities or viral infections; and most of them are acute or subacute onset [1]. It usually occurs in young adults and has a history of respiratory infection or vaccination within 2 weeks before the illness. Cold, overwork, and trauma are

Manuscript submitted February 9, 2019, accepted February 25, 2019

aDepartment of Neurology, Taihe Hospital Affiliated to Hubei University of Medicine, Shiyan City, Hubei Province, China

${ }^{b}$ Corresponding Author: Yi Bao, Department of Neurology, Taihe Hospital Affiliated to Hubei University of Medicine, Shiyan City, Hubei Province, China. Email:karlbaoyi@163.com

doi: https://doi.org/10.14740/jmc3270 common causes. The first symptom is numbness and weakness in both lower extremities, and the condition can reach a peak at 2 - 3 weeks. The pathological changes are characterized by loss of myelin sheath of nerve fibers and relatively complete nerve cells. In severe cases, it can invade the spinal anterior horn cells, brain stem nucleus and brain motor cortical pyramidal cells, so sometimes life-threatening. Typical clinical manifestations include incomplete transverse lesions, unilateral or bilateral weakness or paralysis of lower limbs, accompanied by numbness, no obvious sensory disturbance plane or have two planes, and bladder and rectal dysfunction, a few accompanied by headache [2]. The myelin sheath of acute demyelinating disease can be regenerated; the faster the speed, the more complete the degree. The regenerated myelin sheath is thinner, but generally has little effect on functional recovery. Early onset is usually treated with hormones, gamma globulin and symptomatic support, but it is easy to relapse [3].

This article described a stroke-like onset, which was characterized by a left limb muscle strength presenting as grade 0 , complete paralysis and partial sensory disturbance; and the lesion was in the medulla oblongata and cervical cord junction, which is rare in demyelinating lesions. Initially she was misdiagnosed as stroke, the hormone combined with globulin treatment quickly improved the patient's symptoms and saved limb function. At the second visit, the patient recovered well without recurrence during long-term follow-up. The specific pathogenesis, diagnosis and treatment of the patient are reported as follows.

\section{Case Report}

A 32-year-old woman presented with acute onset of left neck pain after waking up and numbness after left upper limb shaking that rapidly spreaded to the limbs. Subsequently, the left limb was unable to move. There was no diplopia, vomiting, slurred speech, choking cough, unconscious disorder, or convulsion. No hemorrhage in the brain computed tomography (CT) was found in the local hospital. The patient quickly developed urinary retention, and she was admitted to our hospital through emergency. She was previously healthy and had a history of upper respiratory tract infection 1 week before onset. Physical examination included muscle strength level 5 of right upper and lower limbs, normal muscle tone. The muscle 

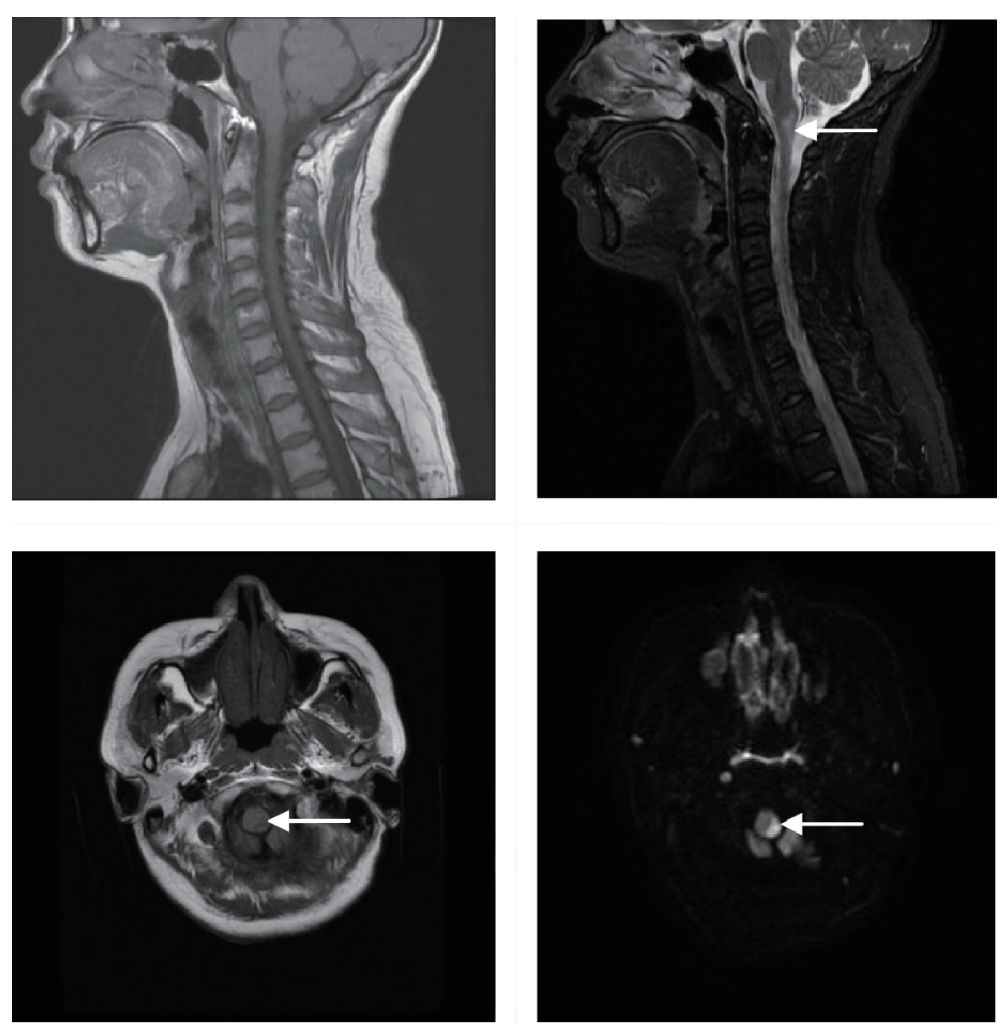

Figure 1. Abnormal signal in the left part of the medulla oblongata and cervical cord junction area.

strength of left upper and lower limbs was level 0 , and the muscle tension was low, left facial palsy decreased, left limb palsy hypersensitivity, right limb palsy decreased, left limb tendinous reflex decreased, the left lower extremity was positive for Babinski sign, the neck was soft and non-resistance, Brudzinski and Kernig signs were negative; and the remaining examination showed no positive signs. The results showed that there were no obvious abnormalities in cerebrospinal fluid (conventional, biochemical, acid-resistant and ink staining, MOG, AQP4, oligoclonal OB, autoimmune encephalitis). To exclude NMDA encephalitis, color Doppler ultrasonography of the uterine accessory showed multiple solid nodules in the uterus (fibroids were considered) and cysts in the left accessory area (chocolate cysts were considered).

The primary diagnosis is that middle-aged woman with acute onset in a quiet state manifested as left hemiplegia after neck pain. Physical examination showed left side of the face tingling sensation decreased, left limb movement and sensation decreased, right limb shallow sensation decreased, left limb pathological sign was positive, consistent with the upper motor neuron paralysis performance. Combined with no evidence of hemorrhage in the brain CT, qualitative diagnosis of ischemic cerebrovascular disease was considered, 11 points according to the NIHSS score, and considered for large vascular type according to the TOAST classification. Positional diagnosis of the responsible vessel being the right middle cerebral artery was considered. The diagnosis of the patients with head and neck pain at the onset of the disease should be differentiated from cerebral hemorrhage; but her CT examination did not support it. It was difficult to explain the pain and urinary retention of the right limb by stoke. In order to trace the etiology, brain MRI examination was done which showed abnormal signal in the left part of the medulla oblongata and cervical cord junction area; so the possibility of demyelinating lesions was high. Brain diffusion-weighted magnetic resonance imaging (DWI) showed that the possibility of demyelinating lesion in the left part of the medulla oblongata and cervical cord junction area was highly likely to have demyelinating lesions. Brain magnetic resonance angiogram (MRA) showed that the A1 segment of the right anterior cerebral artery was slightly thinner. Cervical MRI showed that cervical disc degeneration with mild herniation of c5-6 disc, infarction may occur on the left side of the medulla oblongata and cervical cord junction area. According to these results of magnetic resonance, the demyelinating lesions of the medulla oblongata and cervical cord junction area were diagnosed. See Figure 1 for details.

After the diagnostic using of methylprednisolone (40 mg), the left limb muscle strength of the patient recovered from level 0 to level 2, which proved that the treatment was effective. Chest CT, hip CT, tuberculosis antibody and hepatitis markers were examined to exclude tuberculosis and hepatitis. She was given methylprednisolone $(500 \mathrm{mg})$ shock treatment and gamma globulin (25 g) to clear antibodies; measures to improve the circulation, nerve-nurturing, nutritional support and other symptomatic treatment were also given. Indwelling catheter could improve symptoms of urinary retention, defecation enema could keep stool unobstructed, regular turned-over to prevent pressure sores, and low molecular heparin to prevent 
deep venous thrombosis of the lower extremities. If the patient didn't have respiratory, urinary tract infections and pressure sores, she could recover within 3 months; if there were complications, the recovery time would be prolonged; if respiratory muscles were involved, life-threatening complications could be caused.

During treatment, the patient presented with fever and turbid urine; and the urine routine showed white blood cells $2,912.5 / \mu \mathrm{L}$ and bacteria $56,348.7 / \mu \mathrm{L}$. Urinary tract infection was considered; and the patient recovered after treatment with anti-infective drugs. Half a month later, the left limb muscle strength of the patient returned to grade 4; and there were still pain abnormalities, urinary retention and the right side of the pain reduction improved. Re-examination of magnetic resonance showed that the demyelinating lesion recovered. After discharge, long-term use of mycophenolate mofetil dispersible tablets, prednisone, mecobalamine, and vitamin B1 were prescribed. In addition, gastric-protective drugs and calcium to prevent complications were given to the patients, and she was regular reviewed. When the patient came for reexamination 3 months later, the muscle strength of the left side completely returned to normal, but there was still pain.

\section{Discussion}

Demyelinating disease refers to a group of diseases characterized by nerve myelin loss and relatively mild involvement of neurons and axons. Acute encephalomyelitis belongs to the acquired central nervous system demyelinating disease, which mainly occurs in children and young adults; sporadic cases are common. The first symptom is limb distal symmetry weakness, which quickly aggravates and develops to the proximal end. In severe cases, it can cause paralysis of the respiratory muscles and flaccid paralysis. Sensory dysfunction is characterized by abnormal sensation of the distal part of the limb (gloves and sock-like sensation), muscle tenderness, and often bilateral paralysis of the face nerves. Most patients may have respiratory or gastrointestinal infection symptoms before onset. Characteristics of cerebrospinal fluid examination include normal pressure, white blood cells and protein slightly increased, and normal sugar and chloride content. MRI features multiple long T1 and T2 signals in white matter; electrophysiological examination can assist early diagnosis, but no specificity $[4,5]$.

Stroke is an acute cerebrovascular disease that includes ischemic and hemorrhagic stroke. Ischemic stroke accounts for $60-70 \%$ of the total number of strokes. The onset is rapid, the performance is critical; and there may be hemianopia, hemiplegia, and partial sensation. In severe cases, quadriplegia, cerebral palsy, coma, etc., and even life-threatening complications may occur. It has the characteristics of high morbidity, mortality and disability. Cerebral hemorrhage often occurs in the active state, most patients have headache; the diagnosis is mainly dependent on the brain CT which found high density shadow. Ischemic stroke usually starts in a quiet state without headache. Magnetic resonance imaging (DWI sequence) finding high signal is helpful for early diagnosis $[6,7]$.

The characteristics of this case included acute onset, pre- senting as hemiplegia of the left limb and partial sensory disorder, no hemorrhage observed in brain CT, easily misdiagnosed as acute ischemic stroke. However, the patient had some inconsistency, such as middle-aged female, no history of smoking or drinking, no chronic history of hypertension, diabetes or other diseases, no basis for vascular diseases; and in the meantime, she had a history of upper respiratory tract infection, had superficial hypoesthesia in the right limb and urinary retention, and was found to be consistent with the manifestations of Brown-Sequard syndrome. The patient had a sensory disturbance on the right side of the face, which reminded us the involvement of the trigeminal spinal nucleus. The special feature of this case was that the rate of progression was faster than that of general demyelinating disease, which was inconsistent with the transverse damage that occurred in most acute encephalomyelitis. Brain MRI showed a left abnormal signal in the medulla oblongata and cervical cord junction area. The possibility of demyelinating lesions was great, but cervical MRI diagnosis was a cerebral infarction lesion, which was one of the reasons for misdiagnosis.

At present, there is no effective radical treatment for acute inflammatory demyelinating lesions, and the main purpose of treatment is to inhibit disease progression and avoid recurrence. Corticosteroids, $\beta$-interferon, immunosuppressants, immunoglobulins are commonly used drugs; plasma exchange therapy can also be used; and the combination of corticosteroids and immunoglobulins is more effective. Symptomatic treatment includes alleviating painful phlegm, improving bladder rectal dysfunction, and preventing pressure sores. During bed rest, it is necessary to strengthen nursing and early rehabilitation to keep the airway unobstructed, and tracheotomy or ventilator-assisted breathing should be performed at an early stage if necessary [8]. After diagnosed hormone therapy, the patient's muscle strength was restored from grade 0 to grade 2. After massive hormone therapy combined with intravenous immunoglobulin therapy, the muscle strength returned to normal, but there was still left limb paresthesia. Indwelling catheter catheterization was performed due to urinary retention but urinary tract infection still occurred. Fortunately, in the case of careful nursing and the use of low molecular weight heparin to prevent thrombosis, although the patient had been in bed for half a month, no pulmonary infection, bedsores, or venous thrombosis of lower extremities occurred. After discharge, the patient was taking a small amount of immunomodulatory drugs for a long period of time. During the follow-up visit, the patient recovered well and there was no recurrence during long-term follow-up.

\section{Acknowledgments}

We are grateful to the medical staff who have treated this patient carefully.

\section{Funding Disclosure}

This is a clinical observation article without any funding. 


\section{Conflict of Interest}

The authors declare that they have no conflict of interest.

\section{Informed Consent}

We obtained the patient's informed consent.

\section{Author Contributions}

Yi Bao summed up and wrote the article; Xingyue Li wrote the article; Yajie Hu, Lixia Qin, Miao Zhang, Ying Wang, and Guangjian Liu collected the case data.

\section{References}

1. Mayo L, Quintana FJ, Weiner HL. The innate immune system in demyelinating disease. Immunol Rev. 2012;248(1):170-187.

2. Totaro R, Di Carmine C, Marini C, et al. Tumefactive demyelinating lesions: spectrum of disease, diagnosis and treatment. Curr Neurobiol. 2016;7(1):21-26.

3. Cortese A, Franciotta D, Alfonsi E, Visigalli N, Zardini E, Diamanti L, Prunetti P, et al. Combined central and peripheral demyelination: Clinical features, diagnostic findings, and treatment. J Neurol Sci. 2016;363:182-187.

4. Hardy TA, Reddel SW, Barnett MH, Palace J, Lucchinetti CF, Weinshenker BG. Atypical inflammatory demyelinating syndromes of the CNS. Lancet Neurol. 2016;15(9):967-981.

5. Scheller NM, Svanstrom H, Pasternak B, Arnheim-Dahlstrom L, Sundstrom K, Fink K, Hviid A. Quadrivalent HPV vaccination and risk of multiple sclerosis and other demyelinating diseases of the central nervous system. JAMA. 2015;313(1):54-61.

6. Goyal M, Demchuk AM, Menon BK, Eesa M, Rempel JL, Thornton J, Roy D, et al. Randomized assessment of rapid endovascular treatment of ischemic stroke. N Engl J Med. 2015;372(11):1019-1030.

7. Berkhemer OA, Fransen PS, Beumer D, van den Berg LA, Lingsma HF, Yoo AJ, Schonewille WJ, et al. A randomized trial of intraarterial treatment for acute ischemic stroke. N Engl J Med. 2015;372(1):11-20.

8. Berzero G, Cortese A, Ravaglia S, Marchioni E. Diagnosis and therapy of acute disseminated encephalomyelitis and its variants. Expert Rev Neurother. 2016;16(1):83-101. 